\title{
Diet-induced changes in titer support a threshold effect of Wolbachia-associated plastic recombination in Drosophila melanogaster
}

Sabrina L. Mostoufi, ${ }^{1, *}$ and Nadia D. Singh ${ }^{1, *}$

${ }^{1}$ Department of Biology, University of Oregon, Eugene, OR 97403

\section{*Correspondence}

Sabrina L. Mostoufi: smostouf@uoregon.edu and Nadia D. Singh: nsingh@uoregon.edu

Keywords: Wolbachia, Drosophila melanogaster, plastic recombination, bacterial titer, diet 


\section{ABSTRACT}

2 Plastic recombination in Drosophila melanogaster has been associated with a variety of

3 extrinsic and intrinsic factors such as temperature, starvation, and parasite infection. The

4 bacterial endosymbiont Wolbachia pipientis has also been associated with plastic recombination

5 in D. melanogaster. Wolbachia infection is pervasive in arthropods and this infection induces a

6 variety of phenotypes in its hosts, the strength of which can depend on bacterial concentration, or

7 titer. Here we test the hypothesis that the magnitude of Wolbachia-associated plastic

8 recombination in $D$. melanogaster depends on titer. To manipulate titer, we raised Wolbachia-

9 infected and uninfected flies on diets that have previously been shown to increase or decrease

10 Wolbachia titer relative to controls. We measured recombination in treated and control

11 individuals using a standard backcrossing scheme with two X-linked visible markers. Our results

12 recapitulate previous findings that Wolbachia infection is associated with increased

13 recombination rate across the yellow-vermillion interval of the $\mathrm{X}$ chromosome. Our data show no

14 significant effect of diet or diet by Wolbachia interactions on recombination, suggesting that

15 diet-induced changes in Wolbachia titer have no effect on the magnitude of plastic

16 recombination. These findings represent the first step toward investigating the mechanisms

17 behind Wolbachia-associated plastic recombination and demonstrate that the effect may be

18 threshold-based as opposed to dose-dependent. 


\section{INTRODUCTION}

Phenotypic plasticity is the phenomenon by which a single genotype may produce

21 multiple phenotypes in response to variable environmental stimuli. Plasticity is pervasive in

22 nature, affecting a range of phenotypes like morphology, development, behavior, and

23 reproduction in bacteria, plants, and animals (Fusco and Minelli 2010; Forsman 2015; Fox et al.

24 2018). Meiotic recombination has also been shown to be phenotypically plastic, where the

25 proportion of recombinant offspring increases in response to environmental stimuli. Plastic

26 recombination has been observed in a number of taxa and in response to different stimuli: yeast

27 experience elevated recombination rates under nutrient stress (Abdullah and Borts 2001),

28 Arabidopsis displays recombination plasticity when exposed to extreme temperatures (Francis

29 2007; Saini et al. 2017; Lloyd et al. 2018; Modliszewski et al. 2018), infection causes increased

30 recombination in mosquitoes (Zilio 2018) and plants (Chiriac et al. 2006; Andronic 2012), and

31 social stress is associated with plastic recombination in male mice (Belyaev and Borodin 1982).

32 Plastic recombination also has a rich history of study in the fruit fly, Drosophila

33 melanogaster. Temperature was the first condition associated with plastic recombination in $D$.

34 melanogaster, a phenomenon which has been well-characterized over the last century (Plough

35 1917; Plough 1921; Stern 1926; Hayman 1960; Grell 1978; Kohl and Singh 2018). Several other

36 factors have been identified which induce plastic recombination in D. melanogaster, including

37 maternal age (Bridges 1927; Priest 2007; Hunter et al. 2016a), starvation (Neel 1941), heat shock

38 (Zhong 2011; Jackson et al. 2015), and parasite infection (Singh et al. 2015).

39 More recently, infection with the bacteria Wolbachia pipientis has been associated with

40 plastic recombination in D. melanogaster (Singh 2019). Wolbachia is a Gram-negative

41 endosymbiont that infects between 40-60\% of arthropod species (Zug and Hammerstein 2012). 
42 In the genus Drosophila, Wolbachia primarily inhabits the germ cells and are maternally-

43 inherited through the oocyte (Pietri et al. 2016). Different Drosophila species are infected with

44 unique strains of Wolbachia, each with varied effects on host biology (for review, see Serbus et

45 al. 2008; Correa and Ballard 2016). One of the most well-studied Wolbachia-associated

46 phenotypes is cytoplasmic incompatibility, which causes certain mating pairings between

47 infected and uninfected flies to produce nonviable embryos (Turelli and Hoffmann 1995). Other

48 strains of Wolbachia can cause phenotypes like male offspring killing, parthenogenesis, or

49 decreased lifespan (Hurst et al. 2000; Pietri et al. 2016). The native Wolbachia strain in D.

50 melanogaster, wMel, has been shown to provide protection against viral pathogens (Hedges et

51 al. 2008), increase fecundity in its host (Pietri et al. 2016), and now is associated with plastic

52 increases in recombination rate (Singh 2019).

Since Wolbachia's role in plastic recombination has only recently been discovered, there

54 remains a large gap in our understanding of this interaction. One of the first papers to identify

55 this phenomenon observed a correlation between Wolbachia infection and increased

56 recombination across an interval of the $\mathrm{X}$ chromosome, but not on chromosome 3 (Hunter et al.

57 2016b). This finding was experimentally validated and expanded upon to demonstrate that

58 Wolbachia's effect on recombination was plastic and occurred in multiple strains of $D$.

59 melanogaster (Singh 2019). Yet the scope, magnitude, and mechanisms behind this phenomenon

60 are unclear. Of particular interest is the potential effect of magnitude in Wolbachia-associated

61 plastic recombination. Many factors associated with plastic recombination have been shown to

62 influence the magnitude of increase in recombination rate, depending on the strength of the

63 factor. For example, increased exposure time to heat shock increases the magnitude of plastic

64 recombination in D. melanogaster in a dose-dependent manner (Jackson et al. 2015). This raises 
65 an interesting question of how plastic recombination may be influenced by the strength of

66 Wolbachia infection.

An obvious candidate for testing this question of magnitude is bacterial abundance, or

68 titer. Wolbachia-associated phenotypes can vary according to bacterial titer, including

69 cytoplasmic incompatibility (Calvitti et al. 2015) and viral pathogen protection (Ye et al. 2016).

70 These phenotypes are considered dose-dependent because the strength of the phenotype is

71 correlated with the amount of Wolbachia present within the host. However, some Wolbachia-

72 associated phenotypes instead display a threshold dependency, where the phenotype is not

73 expressed until a certain Wolbachia titer has been reached, as is the case in male-killing (Hurst et

74 al.2000) and lifespan shortening (Reynolds et al. 2003, Chrostek and Teixeira 2018). It is

75 currently unknown what role bacterial titer plays in Wolbachia-associated plastic recombination

76 and whether this phenotype is a result of a threshold level effect, is dose-dependent, or a

77 combination of the two.

78 To address this question, we tested the effect of Wolbachia titer on plastic recombination

79 in D. melanogaster. We used host diet to manipulate Wolbachia titer in fly ovaries under control,

80 yeast-enriched, and sucrose-enriched conditions in order to evaluate the effect of titer on plastic

81 recombination. Recombination rate was measured using classic genetic approaches in

82 Wolbachia-infected and uninfected flies across a genomic interval on the X chromosome. Our

83 data recapitulate that Wolbachia infection is associated with increased recombination rate and

84 suggest that diet-induced changes in titer have no effect on the magnitude of plastic

85 recombination. These findings are among the first to demonstrate that Wolbachia-associated

86 plastic recombination may be a threshold level effect rather than dose-dependent. 
Fly strain and rearing: The $D$. melanogaster strain used in this experiment was RAL306, which

90 comes from the Drosophila Genetics Reference Panel (DGRP) (Mackay et al. 2012; Huang et al.

91 2014). We used the RAL306 strain because it is naturally infected with Wolbachia and exhibits

92 Wolbachia-associated plastic recombination (Hunter et al. 2016b; Singh 2019). To generate

93 uninfected controls, we raised flies on tetracycline-containing media for two generations in order

94 to remove Wolbachia. Tetracycline-containing media was created using standard

95 cornmeal/molasses media containing ethanol-dissolved tetracycline at a final concentration of

$960.25 \mathrm{mg} / \mathrm{mL}$ media (Holden and Jones 1993). Following two generations of tetracycline

97 treatment, flies were then raised on standard media for over ten generations in order to allow the 98 gut microbiome to recolonize the flies (Singh 2019).

We used PCR to confirm Wolbachia infection status prior to the start of the experiment.

100 Briefly, single females were collected from stock vials of Wolbachia-infected and uninfected

101 RAL306 flies. DNA was extracted from these females with a standard squish protocol (Gloor

102 and Engels 1992) and used in PCR with primers for the Wolbachia gene, Wolbachia surface

103 protein (WSP), to identify the presence of Wolbachia (Jeyaprakash and Hoy 2000; Singh 2019).

105 Food treatments: Wolbachia cannot currently be transgenically modified, making it impossible

106 to use genetic engineering to test for differences in titer. Several other factors have been shown

107 to alter Wolbachia titer, including temperature (Hurst et al. 2000; Moghadam et al. 2018),

108 bacterial strain (Chrostek and Teixeira 2018), and host diet (Serbus et al. 2015). However, both

109 temperature and bacterial genotype also affect recombination rate in D. melanogaster. Host diet 
110 has been shown to alter Wolbachia titer within fly ovaries, specifically that a yeast-enriched diet

111 decreases titer while a sucrose-enriched diet increases titer (Serbus et al. 2015). While starvation

112 in larvae is known to alter recombination rate (Neel 1941), there is no evidence that alterations in

113 adult diet affect plastic recombination. Therefore, we used host diet to manipulate Wolbachia

114 titer. For both Wolbachia-infected and uninfected groups, flies were raised on one of three diet

115 treatments: control, yeast-enriched, or sucrose-enriched. We set up ten replicate vials for each

116 experimental group in a single block, repeated for four total blocks.

117 To produce the sucrose-enriched diets, we made a $40 \%$ sucrose mixture following Serbus

118 et al. (2015). Initially, we crossed flies in pure sucrose-enriched vials, but larvae raised on

119 sucrose media showed increased mortality and slower development (unpublished observations).

120 Therefore, we devised a strategy to allow adult flies to feed on the sucrose-enriched media while

121 also promoting normal larval development by using "sucrose patties". Sucrose-enriched mixture

122 was poured into vials and allowed to cool before being sliced into $1 \mathrm{~cm}$ patties, which were

123 placed on top of control food vials. This strategy allowed adult flies to feed on sucrose-enriched

124 food while larvae could burrow down to feed on control food after hatching.

125 To make the yeast-enriched diets, we made a standard yeast paste by mixing dry active

126 yeast and deionized water (Serbus et al. 2015). Approximately $2 \mathrm{~mL}$ of paste was added to

127 control food vials for the yeast-enriched treatments. Similar to the sucrose-enriched patties, this

128 allowed adult flies to feed on yeast-enriched media while larvae could develop on control food.

130 Experimental crosses: Since Wolbachia have been shown to increase recombination on the X

131 chromosome (Singh 2019), we measured recombination with a standard two-step backcrossing

132 scheme using the markers yellow (y) and vermillion (v) (33 cM apart) (Figure 1). In the first 
133 cross, roughly 20 RAL306 females and $20 y v$ males were crossed in 8oz bottles. Heterozygous

134 F1 virgin female offspring were collected from these bottles. For the second cross, 5 females

135 were backcrossed to $5 y v$ males in a vial, with approximately 10 vials per diet treatment per

136 block, repeated for a total of 4 blocks. BC1 offspring (Figure 1) were counted to estimate

137 recombination rate in F1 females by calculating the recombinant fraction, or the proportion of

138 recombinant types to the total number of offspring. For these crosses, recombinant types were

139 heterozygous (female BC1) or hemizygous (male BC1) for either the $y$ or $v$ allele (Figure 1).

$140 \quad$ All crosses were conducted at $25^{\circ} \mathrm{C}$ with a $12: 12$ hour light:dark cycle. Virgins were age-

141 matched at approximately 48 hours before crossing. In each cross, flies were allowed to mate and

142 lay eggs for four days before being removed.

144 Measuring Wolbachia titer: We collected and froze F1 females after egg-laying for whole-body

145 and ovary DNA extraction using the DNeasy® Blood \& Tissue Kit following insect and Gram-

146 negative bacteria protocols (Qiagen, Hilden, Germany). Quantitative PCR (qPCR) was

147 conducted to amplify Wolbachia DNA and estimate the titer within each fly relative to host gene

148 controls (Mouton et al. 2003). We used the SYBR Green Mastermix (Life Technologies,

149 Carlsbad, CA, USA) and standard manufacturer's protocols for qPCR. Results from qPCR are

150 presented as $\mathrm{Cq}$ scores, which represent the number of cycles it takes for each sample to reach a

151 threshold amplification level. Comparing $\mathrm{Cq}$ scores allows us to compare the relative starting

152 amount of product in each sample, where samples with a higher Wolbachia titer should take

153 fewer cycles to amplify and therefore have a lower $\mathrm{Cq}$ score than samples with a lower

154 Wolbachia titer. Both the $\mathrm{Cq}$ scores and $\mathrm{dCq}$ scores were compared between diet treatment 
155 groups; $\mathrm{dCq}$ is calculated by subtracting WSP expression from the mean expression of the host

156 control gene.

158 Statistical analyses: Recombination rate between groups was compared using a logistic

159 regression model to evaluate statistical significance of the effect of Wolbachia infection $\left(W_{j}\right)$,

$160 \operatorname{diet}\left(D_{i}\right)$, or Wolbachia by diet interaction effects $\left(D_{i} \times W_{j}\right)$. The full model is as follows: $Y_{i, j}=$

$161 \mu+D_{i}+W_{j}+D_{i} \times W_{j}+\varepsilon,($ for $i=1 \ldots 3, j=1 \ldots 2)$. We used the statistical software JMP

162 Pro (v14.1.0) for logistic regression modeling, using a general linear model with binomial

163 distribution and link logit function.

$164 \quad$ All other statistical analyses were carried out in R (v1.2.5033). Mutant markers were

165 tested for viability defects using G-tests for goodness of fit. A one-way ANOVA was used to

166 analyze differences in both qPCR results and fly fecundity between experimental groups. A post-

167 hoc analysis of recombination rate variance was conducted using a modified robust Brown-

168 Forsythe Levene-type test and Tukey's multiple comparisons test. The significance threshold for

169 the aforementioned statistical tests was set at 0.05 . Power analyses were conducted using the R

170 package "SIMR" to validate experimental results (Green and MacLeod 2016). Simulated data

171 were generated in $\mathrm{R}$ to produce a range of differences in mean recombination rate between

172 groups, which were tested using repeated simulations in SIMR to calculate statistical power,

173 where $80 \%$ power or greater is considered ideal.

175 Reagent and data availability: Fly strains are available upon request. Raw data will be publicly 176 available prior to publication. 


\section{RESULTS}

178

$194+v$ flies. The null hypothesis is a 1:1 ratio for all phenotypic classes compared. Significant 195 deviations from expected ratios would indicate that the markers affected the viability of certain 196 phenotype combinations, which would negatively impact recombination rate estimates. Similar to previous work (Hunter et al. 2016b, Singh 2019), we find small but

Fly fecundity: In order to assess the effect of Wolbachia titer on plastic recombination, we set up crosses for Wolbachia-infected and uninfected flies on 3 diet treatments and measured recombination between the yellow and vermillion interval on the $\mathrm{X}$ chromosome. In total, 22,228 BC1 flies were scored for recombination (Table 1). For flies fed a control diet, the number of progeny per vial for Wolbachia-infected flies averaged 110 flies/vial, while uninfected flies averaged 111 flies/vial. On a sucrose-enriched diet, Wolbachia-infected flies produced an average of 117 flies/vial, while uninfected flies produced an average of 132 flies/vial. Finally, the number of progeny per vial for flies fed a yeast-enriched diet averaged 225 flies/vial, while uninfected flies averaged 208 flies/vial (Figure 2). Results from a one-way ANOVA test demonstrated that diet treatment $(P<2 \mathrm{e}-16$, ANOVA $(\mathrm{N}=150, \mathrm{df}=2))$, but not Wolbachia infection $(P=0.942$, ANOVA $(\mathrm{N}=150, \mathrm{df}=1))$ significantly affected fly fecundity.

Viability effects of mutant markers: To determine whether the viability of the mutant markers affected the ratios of offspring phenotypes, we performed G-tests for goodness of fit within each vial for the following ratios: males vs. females, wildtype $(++)$ flies vs. $y v$ flies, and $y+$ flies vs. nonsignificant viability defects associated with these markers. Out of 151 crosses, seven showed significant deviation with regards to the male-female ratio, eleven deviated from expected 
wildtype to $y v$ ratios, and nine deviated from the expected ratio of $y+$ to $+v$ flies. However, after

201 using the Bonferroni correction for multiple tests, only one of the deviant crosses remained

202 significant $(P=1.14 \mathrm{E}-12$, G-test $)$. This specific cross had a ratio of 9.8 wildtype flies to $y v$ flies

203 and a recombinant fraction of 0.05. As this likely stems from mating contamination, we

204 discarded this particular cross from further analyses.

205

206 The effect of infection and diet on recombination: We used logistic regression modeling to

207 identify variables which significantly contributed to differences in mean recombination rate

208 between experimental groups. Results are shown in Table 2, where Wolbachia infection $(P=$

$2090.0008, X^{2}$ test $\left.(\mathrm{N}=150, \mathrm{df}=1)\right)$ and experimental block $\left(P=0.0001, X^{2}\right.$ test $\left.(\mathrm{N}=150, \mathrm{df}=3)\right)$

210 were significantly associated with differences in recombination rate. The effect of Wolbachia

211 infection can be seen clearly in Figure 3, where Wolbachia-infected flies display an average

212 increase of $2.4 \mathrm{cM}$ in recombination rate over uninfected flies in each diet treatment. Neither

213 host diet $\left(P=0.42, X^{2}\right.$ test $\left.(\mathrm{N}=150, \mathrm{df}=2)\right)$ nor infection by diet interaction effects $\left(P=0.43, X^{2}\right.$

214 test $(\mathrm{N}=150, \mathrm{df}=2))$ was significant. Based on the power of our tests, we would have been able

215 to detect a difference of $5.8 \%$ or greater between group means, which corresponds to a difference

216 in recombination rate of approximately $2 \mathrm{cM}$ (Figure S1). This indicates that the effect of diet or

217 Wolbachia titer, if present, was weaker than the effect of Wolbachia infection alone.

218 We also tested for the effect of Wolbachia infection, titer, and diet on recombination rate

219 variance, which was calculated as absolute residuals. Uninfected flies showed no significant

220 difference in recombination rate variance between diet treatment groups $(P=0.25$, Levene's test

$221(\mathrm{~N}=75, \mathrm{df}=2))$, and a comparison between uninfected and Wolbachia-infected flies was also

222 nonsignificant $(P=0.11$, Levene's test $(\mathrm{N}=150, \mathrm{df}=1))$. However, Wolbachia-infected flies 
223 displayed significant differences in variance between diet treatment groups $(P=0.007$, Levene's

224 test $(\mathrm{N}=75, \mathrm{df}=2))$ and a Tukey's multiple comparisons test found that infected flies on a

225 sucrose-enriched diet were significantly different from flies on a control $(P=0.03)$ and yeast-

226 enriched $\operatorname{diet}(P=0.003)$.

228 Host diet and quantitative PCR: In order to compare Wolbachia titer between diet treatment

229 groups, DNA was extracted from female F1 flies after crossing. Results from ovary samples are

230 shown in Figure 4, where Cq scores are presented for WSP expression in both ovary and whole-

231 body samples of Wolbachia-infected flies. Uninfected flies showed undetermined Cq scores for

232 WSP, as expected for flies lacking Wolbachia (data not shown). Cq scores for WSP expression

233 were significantly different between diet treatment groups for both whole-body $(P=8.64 \mathrm{e}-05$,

234 ANOVA $(\mathrm{N}=36, \mathrm{df}=2))$ and ovarian samples $(P=1.76 \mathrm{e}-07$, ANOVA $(\mathrm{N}=22, \mathrm{df}=2))$.

235 However, these differences did not follow expected Wolbachia titer levels for each diet

236 treatment, particularly for the yeast-enriched diet treatment. There were no significant

237 differences in $\mathrm{dCq}$ scores for WSP expression between diet treatment groups for either whole-

238 body $(P=0.388$, ANOVA $(\mathrm{N}=36, \mathrm{df}=2))$ or ovarian samples $(P=0.193$, ANOVA $(\mathrm{N}=22, \mathrm{df}$

$239=2))($ Figure S2). 


\section{DISCUSSION}

242 Effect of Wolbachia infection and diet on recombination: The goal of this experiment was to

243 assess whether Wolbachia-associated plastic recombination in D. melanogaster is dose-

244 dependent or threshold-dependent. To address that question, we tested the effects of Wolbachia

245 infection, host diet, and Wolbachia titer on recombination rate. We find that Wolbachia infection

246 is associated with a significant increase in recombination rate on the $\mathrm{X}$ chromosome (Table 2).

247 Our data indicate that the Wolbachia-associated increase in recombination is robust with regards

248 to variation in host diet, as Wolbachia-infected flies displayed a higher recombination rate than

249 their uninfected counterparts in each diet treatment (Figure 3). This finding adds to a growing

250 body of literature which supports Wolbachia as an inducer of plastic recombination in $D$.

251 melanogaster (Hunter et al. 2016b; Singh 2019; Bryant and Newton 2020).

252 We also tested the effect of host diet on plastic recombination, where we find that diet

253 had no effect on recombination rate in Wolbachia-uninfected flies (Table 2, Figure 3). This result

254 contrasts previous findings which reported that starvation in larvae was associated with increased

255 recombination rate (Neel 1941). Differences between our study and the previous one may

256 indicate that only severe changes in diet such as starvation are sufficient to induce plastic

257 recombination in D. melanogaster. However, it should also be noted that Neel's study was

258 carried out using markers on chromosome 3 (1941) while our study assessed recombination on

259 the X chromosome. This may suggest that diet-associated plastic recombination is variable

260 across the genome, as is the case for other conditions associated with plastic recombination such

261 as temperature and Wolbachia infection (Grell 1978; Singh 2019). Outside of the present study,

262 no recent investigations have been made into how starvation or diet affects recombination in 
263 flies, highlighting a need for additional research into the role diet may play in plastic

264 recombination.

Though diet did not affect recombination rate, there was an effect of diet on fecundity.

266 We observed that the average number of offspring per vial was significantly different between

267 the diet treatments, with yeast-fed flies displaying the highest average fecundity (Figure 2). The

268 influence of diet on lifespan and fecundity in D. melanogaster has been well-characterized,

269 especially regarding sucrose and yeast content (Drummond-Barbosa and Spradling 2001; Bass et

270 al.2007). Specifically concerning fecundity, yeast-enriched diets greatly increase female

271 fecundity, while sucrose-enriched diets decrease female fecundity (Bass et al. 2007).

273 (Weeks et al. 2007; Mazzetto et al. 2015; Singh 2019), we found no significant effect of

274 Wolbachia infection on fecundity. However, this may reflect a strain-specific response, rather

275 than the effect of Wolbachia infection on D. melanogaster as a whole. Differences in fecundity

276 between flies depends on Wolbachia genotype (Gruntenko et al. 2019), host genotype (Fry et al.

277 2004), and bacterial-host interactions (Singh 2019). For instance, the strain used in this

278 experiment, RAL306, was also used in a study which reported an overall effect of Wolbachia

279 infection on fecundity across multiple strains (Singh 2019). However, when examined

280 individually, Wolbachia-infected RAL306 flies had a lower mean fecundity than uninfected

281 RAL306 flies (Singh 2019). This suggests that Wolbachia broadly impacts fecundity, but this

282 effect may vary with host genotype.

284 Effect of Wolbachia titer on recombination rate: By using host diet to manipulate Wolbachia

285 titer (Serbus et al. 2015), we aimed to test the effect of titer on the magnitude of Wolbachia- 
associated plastic recombination. Though we expected qPCR Cq scores to correlate with

predicted Wolbachia titer for each diet treatment, our results did not follow expected differences in WSP abundance between groups. While we expected a yeast-enriched diet to decrease

289 Wolbachia titer and be reflected in a higher Cq score compared to control flies, yeast-fed flies

290 instead had low Cq scores similar to sucrose-fed flies, which would indicate that yeast-fed flies

291 had a high Wolbachia titer and contradicts previous findings (Serbus et al. 2015; Christensen et

292 al. 2019). We can interpret these results in one of two ways: either qPCR did not accurately

293 capture differences in Wolbachia titer, or host diet does not reliably influence Wolbachia titer.

294 Though papers have successfully measured both relative and absolute Wolbachia

295 abundance in D. melanogaster (Newton et al. 2015; Christensen et al. 2019), others have found

296 qPCR results to be ineffective or misleading when measuring ovarian Wolbachia titer (Ponton et

297 al. 2014). However, staining and imaging of ovaries has reliably and consistently supported an

298 influence of host diet on Wolbachia titer in D. melanogaster ovaries (Serbus et al. 2015;

299 Camacho et al. 2017; Christensen et al. 2019). If we reason that the uncertainty lies with the

300 technique and not the biological principle, then we can assume qPCR results are inaccurate but

301 Wolbachia titer was changed as expected between diet treatment groups. Combined with the

302 recombination analysis which found no effect of infection by diet interactions (Table 2), these

303 results suggest that Wolbachia titer had no effect on the magnitude of recombination rate

304 increase. Therefore, our experiments find that Wolbachia-associated plastic recombination is not

305 dose-dependent and instead may be caused by a threshold-level effect, which is discussed further

306 below.

However, while Wolbachia titer did not affect the magnitude of recombination, it did

308 influence recombination rate variance. Wolbachia-infected flies fed a sucrose-enriched diet, in 
309 order to promote high Wolbachia titer, had significantly greater variance than Wolbachia-

310 infected flies on either a control or yeast-enriched diet. This finding suggests that increased

311 Wolbachia titer may increase recombination rate variation, rather than increase the average rate

312 of recombination beyond that caused by standard Wolbachia infection. Changes in variance have

313 not previously been reported for other inducers of plastic recombination in D. melanogaster, nor

314 for other Wolbachia-associated host phenotypes, suggesting that this may be a unique feature of

315 Wolbachia-associated plastic recombination. This finding inspires multiple questions for future

316 research, including why low Wolbachia titer did not result in decreased variance and whether

317 this phenomenon is robust in response to other modifiers of Wolbachia titer.

319 Threshold-level effect: Regardless of changes in recombination rate variation, Wolbachia titer

320 did not influence the average rate of recombination in this study, consistent with a threshold

321 effect rather than a dose-dependent model. If Wolbachia-associated plastic recombination is

322 caused by a threshold-level effect, this follows the same trend as male-killing, another

323 Wolbachia-driven trait in Drosophila. In D. bifasciata, Wolbachia infection causes increased

324 mortality of male offspring, leading to modified sex ratios (Hurst et al. 2000). However,

325 Wolbachia titer decreases in flies exposed to elevated temperatures, which causes male mortality

326 rates to decrease and offspring sex ratios to return to normal (Hurst et al. 2000). These findings

327 suggested that this Wolbachia-associated phenotype requires a threshold-level of bacteria in

328 order to be expressed, after which the strength of the phenotype scales with Wolbachia titer and

329 is dose-dependent. The same may be true for Wolbachia-associated plastic recombination, where

330 recombination is modified in infected flies when bacterial titer reaches a threshold level. It may 
331 also be true that Wolbachia-associated plastic recombination is dose-dependent, but only at titer

332 levels more extreme than could be achieved through manipulations in host diet.

333 In contrast to our findings, Bryant and Newton (2020) suggest that Wolbachia-associated

334 plastic recombination is dose-dependent. They find that D. melanogaster infected with the

335 Wolbachia strain wMelPop display a higher recombination rate across the yellow-vermillion

336 interval of the X chromosome when compared to flies infected with a different Wolbachia strain,

337 wMel (Bryant and Newton 2020). The wMelPop strain maintains a higher titer in flies, which

338 would suggest that the magnitude of recombination corresponded with Wolbachia titer and

339 indicates a dose-dependent relationship. Yet it is important to note that this study cannot separate

340 the effect of titer from Wolbachia strain; while wMel is the native Wolbachia strain in D.

341 melanogaster, wMelPop is considered pathogenic because it maintains a high titer and

342 significantly decreases host lifespan (Strunov et al. 2013; Chrostek and Teixeira 2018). Other

343 pathogenic bacteria have been shown to plastically increase recombination rate in $D$.

344 melanogaster (Singh et al. 2015), making it difficult to say whether an increase in recombination

345 rate in wMelPop-infected flies is due to bacterial titer, its pathogenic nature, additional genetic

346 differences between the two bacterial strains, or a combination of factors.

348 The Drosophila microbiome: It may also be true that Wolbachia-associated plastic

349 recombination is dose-dependent, but that this effect is masked in our study due to complex

350 interactions between diet, host, and the microbiome. Diet is known to have a significant impact

351 on microbiome composition in a number of species (Turnbough et al. 2008; Read and Holmes

352 2017; Erkosar et al. 2018). In D. melanogaster, diets rich in either yeast or sucrose caused

353 significant changes in abundance of certain members of the gut microbiome (Chandler et al. 
354 2011). These changes in microbiome composition can have drastic impacts on host biology

355 including hormone production, metabolism, and nutrient acquisition (Leulier et al. 2017).

356 Specific members of the D. melanogaster microbiome have been shown to support larval feeding

357 under starvation conditions (Consuegra et al. 2020), suggesting that diet-induced changes in the

358 microbiome can significantly impact host development. Though our results suggest that changes

359 in diet had no significant effect on recombination rate, as uninfected flies showed similar mean

360 recombination rate for each diet treatment (Figure 3), it is difficult to rule out without directly

361 measuring changes in microbiome composition.

362 In addition to the gut microbiome, which comes in direct contact with nutritional

363 elements, host diet also affects Wolbachia. One finding our study takes advantage of is that

364 increased sucrose or yeast in D. melanogaster diets can manipulate Wolbachia titer (Serbus et al.

365 2015). Wolbachia rely on their host to acquire nutrients, so changes in diet can affect microbe

366 behavior and replication and may ultimately impact host biology. Wolbachia-infected $D$.

367 melanogaster have been shown to alter behavior and diet preference, potentially as a strategy to

368 reduce negative effects on lifespan and fecundity (Ponton et al. 2014; Truitt et al. 2018). Though

369 we saw no significant differences in fecundity or viability related to infection status in this study,

370 it is unclear whether Wolbachia-infected flies fed experimental food experienced changes in

371 behavior or feeding that may have impacted recombination estimates.

372 Finally, the gut microbiome and Wolbachia have been shown to influence one another.

373 Wolbachia infection can alter relative abundances of members of the gut microbiome compared

374 to uninfected flies (Simhadri et al. 2017). Conversely, ingestion of certain species of gut bacteria

375 has been shown to influence Wolbachia abundance (Rudman et al. 2019). Taken together, all of

376 these findings present a complex web of interactions between host, diet, the gut microbiome, and 
377 Wolbachia. Though it is difficult to estimate the impact of these interactions on our results, it

378 remains clear that Wolbachia-associated plastic recombination is robust in response to both

379 measured changes in diet and unmeasured changes in microbiome composition. Future work

380 may focus on studying Wolbachia-only experimental flies, where germ-free flies are reinfected

381 with Wolbachia, in order to remove potentially confounding variables caused by these complex

382 interactions. However, there is also value in studying these systems in their natural state in order

383 to gain a more complete understanding of host-microbe associations.

385 Conclusions: Our current inability to transgenically modify Wolbachia makes it impossible to 386 assess the effect of titer alone on Wolbachia-associated phenotypes. Though differences in titer 387 can be assessed through manipulation of host diet (Serbus et al. 2015), temperature (Hurst et al.

388 2000; Moghadam et al. 2018), or Wolbachia strain (Chrostek and Teixeira 2018), these methods

389 include confounding variables which make it difficult to definitively assign Wolbachia titer as

390 the causative agent in phenotypes of interest. Our present study controls for host and microbe

391 genotype and finds that Wolbachia-associated plastic recombination is a phenotype with a

392 threshold-level effect, while acknowledging the ways in which changes in host diet may

393 influence that finding. Future advances toward making genetic manipulation possible in

394 Wolbachia would allow the role of titer to be more definitively tested without confounding 395 effects.

\section{ACKNOWLEDGEMENTS}

398 We would like to thank the Singh lab group for help with fly counting and feedback on the

399 manuscript, and Daniel T. Grimes for the use of his equipment for qPCR analyses. 


\section{$401 \quad$ FUNDING}

402 This work was supported by the National Institute of General Medical Sciences

403 of the National Institutes of Health under award number [T32GM007413-43] to SLM.

404

\section{CONFLICTS OF INTEREST}

406 The authors declare no conflicts of interest. 


\section{REFERENCES}

408 Abdullah, M. F. F., and R. H. Borts. 2001. Meiotic recombination frequencies are affected by nutritional states in Saccharomyces cerevisiae. Proceedings of the National Academy of

Andronic, L. 2012. Viruses as triggers of DNA rearrangements in host plants. Canadian Journal of Plant Science 92:1083-1091.

413 Bass, T. M., R. C. Grandison, R. Wong, P. Martinez, L. Partridge, and M. D. W. Piper. 2007.

414 Optimization of dietary restriction protocols in Drosophila. Journals of Gerontology - Series

415 A Biological Sciences and Medical Sciences 62:1071-1081.

416 Belyaev, D. K., and P. M. Borodin. 1982. The Influence of Stress on Variation and Its Role in $417 \quad$ Evolution. Biologisches Zentralblatt 101:705-714.

418 Bridges, C. B. 1927. The relation of the age of the female to crossing over in the third 419 chromosome of Drosophila melanogaster. The Journal of General Physiology 8:689-700.

420 Bryant, K. N., and I. L. G. Newton. 2020. The Intracellular Symbiont Wolbachia pipientis

421 Enhances Recombination in a Dose-Dependent Manner. Insects 11:284.

422 Calvitti, M., F. Marini, A. Desiderio, A. Puggioli, and R. Moretti. 2015. Wolbachia Density and 423 Cytoplasmic Incompatibility in Aedes albopictus: Concerns with Using Artificial $424 \quad$ Wolbachia Infection as a Vector Suppression Tool. PLoS ONE 10:e0121813.

425 Camacho, M., M. Oliva, and L. R. Serbus. 2017. Dietary saccharides and sweet tastants have 426 differential effects on colonization of Drosophila oocytes by Wolbachia endosymbionts. 427 Biology Open 6:1074-1083. 
428 Chandler, J. A., J. Lang, S. Bhatnagar, J. A. Eisen, and A. Kopp. 2011. Bacterial communities of

429 diverse Drosophila species: Ecological context of a host-microbe model system. PLoS

$430 \quad$ Genetics 7.

431 Chiriac, G. I., L. I. Andronic, V. V. Bujoreanu, and L. I. Marii. 2006. Features of crossing-over

432 in virus-infected tomato. Central European Journal of Biology 1:386-398.

433 Christensen, S., M. Camacho, Z. Sharmin, A. J. M. Z. Momtaz, L. Perez, et al. 2019.

434 Quantitative methods for assessing local and bodywide contributions to Wolbachia titer in

435 maternal germline cells of Drosophila. BMC Microbiology 19.

436 Chrostek, E., and L. Teixeira. 2018. Within host selection for faster replicating bacterial

437 symbionts. PLoS ONE 13(1):e0191530.

438 Consuegra, J., T. Grenier, P. Baa-Puyoulet, I. Rahioui, H. Akherraz, et al. 2020. Drosophila-

439 associated bacteria differentially shape the nutritional requirements of their host during

$440 \quad$ juvenile growth. PLoS Biology 18:e3000681.

441 Correa, C. C., and J. W. O. Ballard. 2016. Wolbachia associations with insects: Winning or

442 losing against a master manipulator. Frontiers in Ecology and Evolution 3:153.

443 Drummond-Barbosa, D., and A. C. Spradling. 2001. Stem cells and their progeny respond to

444 nutritional changes during Drosophila oogenesis. Developmental Biology 231:265-278.

445 Erkosar, B., E. Yashiro, F. Zajitschek, U. Friberg, A. A. Maklakov, et al. 2018. Host diet

446 mediates a negative relationship between abundance and diversity of Drosophila gut

447 microbiota. Ecology and Evolution 8:9491-9502.

448 Forsman, A. 2015. Rethinking phenotypic plasticity and its consequences for individuals,

$449 \quad$ populations and species. Heredity 115:276-284. 
Fox, R. J., J. M. Donelson, C. Schunter, T. Ravasi, and J. D. Gaitán-Espitia. 2018. Beyond buying time: the role of plasticity in phenotypic adaptation to rapid environmental change. Philosophical Transactions of the Royal Society B: Biological Sciences 374:20180174.

453 Francis, K. E., S. Y. Lam, B. D. Harrison, A. L. Bey, L. E. Berchowitz, and G. P. Copenhaver. 2007. Pollen tetrad-based visual assay for meiotic recombination in Arabidopsis. Proceedings of the National Academy of Sciences of the United States of America

Fry, A. J., M. R. Palmer, and D. M. Rand. 2004. Variable fitness effects of Wolbachia infection in Drosophila melanogaster. Heredity 93:379-389.

Fusco, G., and A. Minelli. 2010. Phenotypic plasticity in development and evolution: facts and concepts. Philosophical Transactions of the Royal Society B: Biological Sciences 365:547-

Gloor, G. and Engels, W.R. 1992. Gloor: Single-fly DNA preps for PCR. Dros. Info. Ser.

463 Green, P., and C. J. Macleod. 2016. SIMR: an R package for power analysis of generalized linear mixed models by simulation. Methods in Ecology and Evolution 7:493-498.

465 Grell, R. F. 1978. A comparison of heat and interchromosomal effects on recombination and interference in Drosophila melanogaster. Genetics 89:65-77.

467 Gruntenko, N. E., E. K. Karpova, N. V. Adonyeva, O. V. Andreenkova, E. V. Burdina, et al. 2019. Drosophila female fertility and juvenile hormone metabolism depends on the type of Wolbachia infection. Journal of Experimental Biology 222.

470 Hayman, D. L., and P. A. Parsons. 1960. The effect of temperature, age and an inversion on 471 recombination values and interference in the X-chromosome of Drosophila melanogaster. $472 \quad$ Genetica 32:74-88. 
473 Hedges, L. M., J. C. Brownlie, S. L. O’Neill, and K. N. Johnson. 2008. Wolbachia and virus

$474 \quad$ protection in insects. Science 322:702.

475 Holden, P. R., P. Jones, and J. F. Y. Brookfield. 1993. Evidence for a Wolbachia symbiont in

476 Drosophila melanogaster. Genet. Res., Camb 62:23-29.

477 Huang, W., A. Massouras, Y. Inoue, J. Peiffer, M. Ràmia, et al. 2014. Natural variation in

478 genome architecture among 205 Drosophila melanogaster Genetic Reference Panel lines.

$479 \quad$ Genome Research 24:1193-1208.

480 Hunter, C. M., M. C. Robinson, D. L. Aylor, and N. D. Singh. 2016a. Genetic background,

481 maternal age, and interaction effects mediate rates of crossing over in Drosophila

482 melanogaster females. G3: Genes, Genomes, Genetics 6:1409-1416.

483 Hunter, C. M., W. Huang, T. F. C. Mackay, and N. D. Singh. 2016b. The Genetic Architecture of

484 Natural Variation in Recombination Rate in Drosophila melanogaster. PLoS Genet

$485 \quad 12: 1005951$.

486 Hurst, G. D. D., A. P. Johnson, J. Hinrich, G. V. D. Schulenburg, and Y. Fuyama. 2000. Male-

487 Killing Wolbachia in Drosophila: A Temperature-Sensitive Trait With a Threshold

$488 \quad$ Bacterial Density. Genetics 156:699-709.

489 Jackson, S., D. M. Nielsen, and N. D. Singh. 2015. Increased exposure to acute thermal stress is

490 associated with a non-linear increase in recombination frequency and an independent linear

491 decrease in fitness in Drosophila. BMC Evolutionary Biology 15:175.

492 Jeyaprakash, A., and M. A. Hoy. 2000. Long PCR improves Wolbachia DNA amplification: wsp

493 sequences found in 76\% of sixty-three arthropod species. Insect Molecular Biology 9:393-

$494 \quad 405$. 
Kohl, K. P., and N. D. Singh. 2018. Experimental evolution across different thermal regimes yields genetic divergence in recombination fraction but no divergence in temperature associated plastic recombination. Evolution 72:989-999.

Leulier, F., L. T. MacNeil, W. Lee, J. F. Rawls, P. D. Cani, M. Schwarzer, L. Zhao, and S. J. Simpson. 2017. Integrative Physiology: At the Crossroads of Nutrition, Microbiota, Animal Physiology, and Human Health. Cell Metabolism 25:522-534.

Lloyd, A., C. Morgan, F. C. H. Franklin, and K. Bomblies. 2018. Plasticity of meiotic recombination rates in response to temperature in Arabidopsis. Genetics 208:1409-1420.

Mackay, T. F. C., S. Richards, E. A. Stone, A. Barbadilla, J. F. Ayroles, et al. 2012. The Drosophila melanogaster Genetic Reference Panel. Nature 482:173-178.

Mazzetto, F., E. Gonella, and A. Alma. 2015. Wolbachia infection affects female fecundity in Drosophila suzukii. Bulletin of Insectology 68:153-157.

Modliszewski, J. L., H. Wang, A. R. Albright, S. M. Lewis, A. R. Bennett, et al. 2018. Elevated temperature increases meiotic crossover frequency via the interfering (Type I) pathway in Arabidopsis thaliana. PLoS Genetics 14:1-15.

Moghadam, N. N., P. M. Thorshauge, T. N. Kristensen, N. de Jonge, S. Bahrndorff, H. Kjeldal, and J. L. Nielsen. 2018. Strong responses of Drosophila melanogaster microbiota to developmental temperature. Fly 12:1-12.

Neel, J. V. 1941. A relation between larval nutrition and the frequency of crossing over in the third chromosome of Drosophila melanogaster. Genetics 26:506-516.

Newton, I. L. G., O. Savytskyy, and K. B. Sheehan. 2015. Wolbachia Utilize Host Actin for Efficient Maternal Transmission in Drosophila melanogaster. PLOS Pathogens 11:e1004798. 
Pietri, J. E., H. DeBruhl, and W. Sullivan. 2016. The rich somatic life of Wolbachia.

$$
\text { MicrobiologyOpen 5:923-936. }
$$

520 Plough, H. H. 1917. The effect of temperature on crossingover in Drosophila. Journal of

$521 \quad$ Experimental Biology 24:147-189.

522 Plough, H. H. 1921. Further studies on the effect of temperature on crossing over. The Journal of $523 \quad$ Experimental Zoology 32:187-202.

524 Ponton, F., K. Wilson, A. Holmes, D. Raubenheimer, K. L. Robinson, and S. J. Simpson. 2014.

525 Macronutrients mediate the functional relationship between Drosophila and Wolbachia.

526 Proceedings of the Royal Society B: Biological Sciences 282:20142029.

527 Priest, N. K., D. A. Roach, and L. F. Galloway. 2007. Mating-induced recombination in fruit $528 \quad$ flies. Evolution 61:160-167.

529 Read, M. N., and A. J. Holmes. 2017. Towards an Integrative Understanding of Diet-Host-Gut $530 \quad$ Microbiome Interactions. Front. Immunol 8:538.

531 Reynolds, K. T., L. J. Thomson, and A. A. Hoffmann. 2003. The Effects of Host Age, Host 532 Nuclear Background and Temperature on Phenotypic Effects of the Virulent Wolbachia 533 Strain popcorn in Drosophila melanogaster. Genetics 164:1027-1034.

534 Rudman, S. M., S. Greenblum, R. C. Hughes, S. Rajpurohit, O. Kiratli, et al. 2019. Microbiome 535 composition shapes rapid genomic adaptation of Drosophila melanogaster. Proceedings of 536 the National Academy of Sciences of the United States of America 116:20025-20032.

537 Saini, R., A. K. Singh, S. Dhanapal, T. H. Saeed, G. J. Hyde, and R. Baskar. 2017. Brief 538 temperature stress during reproductive stages alters meiotic recombination and somatic 539 mutation rates in the progeny of Arabidopsis. BMC Plant Biology 17:1-11. 
Serbus, L. R., P. M. White, J. P. Silva, A. Rabe, L. Teixeira, R. Albertson, and W. Sullivan.

Simhadri, R. K., E. M. Fast, R. Guo, M. J. Schultz, N. Vaisman, L. Ortiz, J. Bybee, B. E. Slatko, Modified by the Endosymbiont Wolbachia. mSphere 2:2:e00287-17.

Singh, N. D. 2019. Wolbachia infection associated with increased recombination in Drosophila. G3: Genes, Genomes, Genetics 9:229-237.

548 Singh, N. D., D. R. Criscoe, S. Skolfield, K. P. Kohl, E. S. Keebaugh, and T. A. Schlenke. 2015.

549 Fruit flies diversify their offspring in response to parasite infection. Science 349:747-750.

550 Stern, C. 1926. An effect of temperature and age on crossing-over in the first chromosome of

551 Drosophila melanogaster. Proc. Natl. Acad. Sci. 12:530-532.

552 Strunov, A., E. Kiseleva, and Y. Gottlieb. 2013. Spatial and temporal distribution of pathogenic

$553 \quad$ Wolbachia strain wMelPop in Drosophila melanogaster central nervous system under

554 different temperature conditions. Journal of Invertebrate Pathology 114:22-30.

555 Truitt, A. M., M. Kapun, R. Kaur, and W. J. Miller. 2018. Wolbachia modifies thermal

556 preference in Drosophila melanogaster. Environmental Microbiology 21:3259-3268.

557 Turelli, M., and A. A. Hoffmann. 1995. Cytoplasmic Incompatibility in Drosophila simulans:

558 Dynamics and Parameter Estimates from Natural Populations. Genetics 140:1319-1338.

559 Ye, Y. H., A. M. Carrasco, Y. Dong, C. M. Sgrò, and E. A. Mcgraw. 2016. The Effect of

560 Temperature on Wolbachia-Mediated Dengue Virus Blocking in Aedes aegypti. Am. J.

$561 \quad$ Trop. Med. Hyg 94:812-819. 
562 Zhong, W., and N. K. Priest. 2011. Stress-induced recombination and the mechanism of

563 evolvability. Behavioral Ecology and Sociobiology 65:493-502.

564 Zilio, G., L. Moesch, N. Bovet, A. Sarr, and J. C. Koella. 2018. The effect of parasite infection

565 on the recombination rate of the mosquito Aedes aegypti. PLoS ONE 13:e0203481.

566 Zug, R., and P. Hammerstein. 2012. Still a Host of Hosts for Wolbachia $\square$ : Analysis of Recent

567 Data Suggests That 40\% of Terrestrial Arthropod Species Are Infected. PLoS ONE

$568 \quad 7: 38544$. 


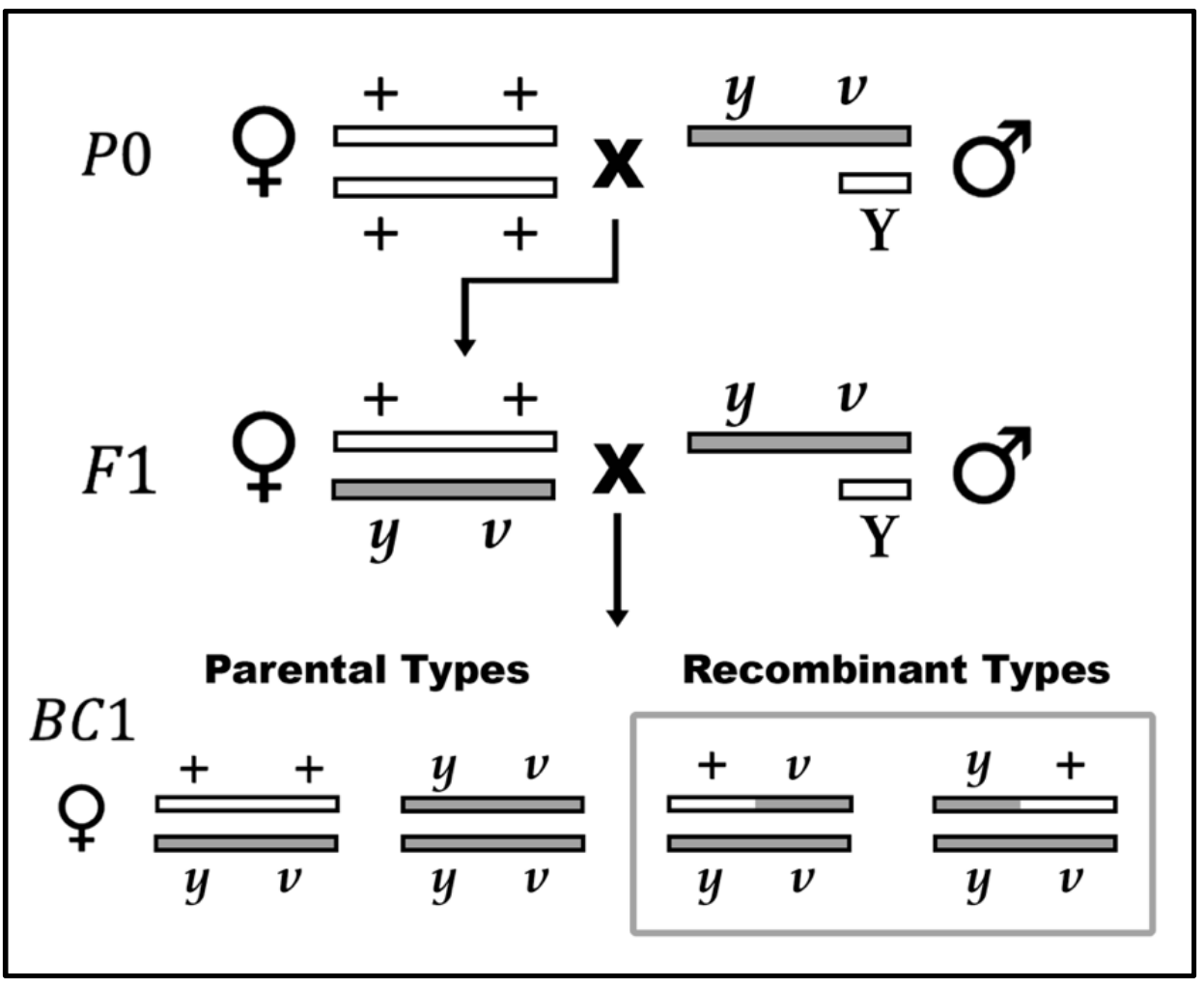

571 Figure 1: A two-step crossing scheme to measure recombination. Recombination rate can be

572 estimated on the $\mathrm{X}$ chromosome using the visual markers yellow (y) and vermillion (v). Males

573 with the $y v$ markers are crossed to wildtype females. Heterozygous F1 females are backcrossed

574 to the same male strain to produce BC1 progeny. Progeny which display either the $y$ or $v$

575 phenotype are considered recombinant. Male BC1 genotypes are not shown, but males are

576 heterogametic and require only one copy of the $y$ or $v$ marker to display a phenotype. 


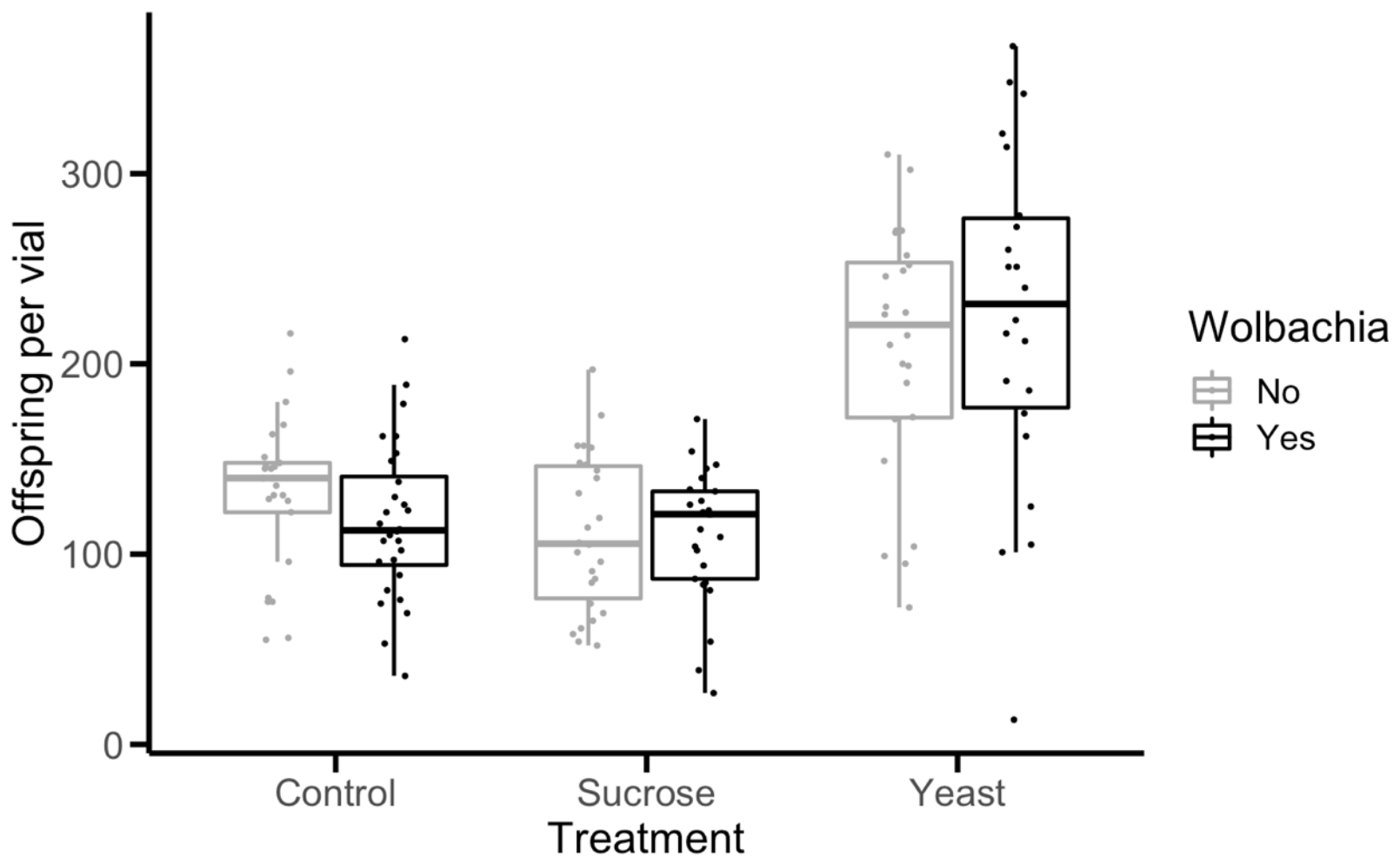

578 Figure 2: Fecundity, or number of offspring per vial, of experimental groups. Wolbachia-

579 infected flies are shown in black, while uninfected flies are shown in gray. Each point

580 corresponds to the total number of offspring in a single vial. Boxplots present summary

581 statistics, where the top and bottom edges encompass the first to third quartiles and the middle

582 bar represents the median for each group. Boxplot whiskers extend to the smallest and largest

583 nonoutliers. 


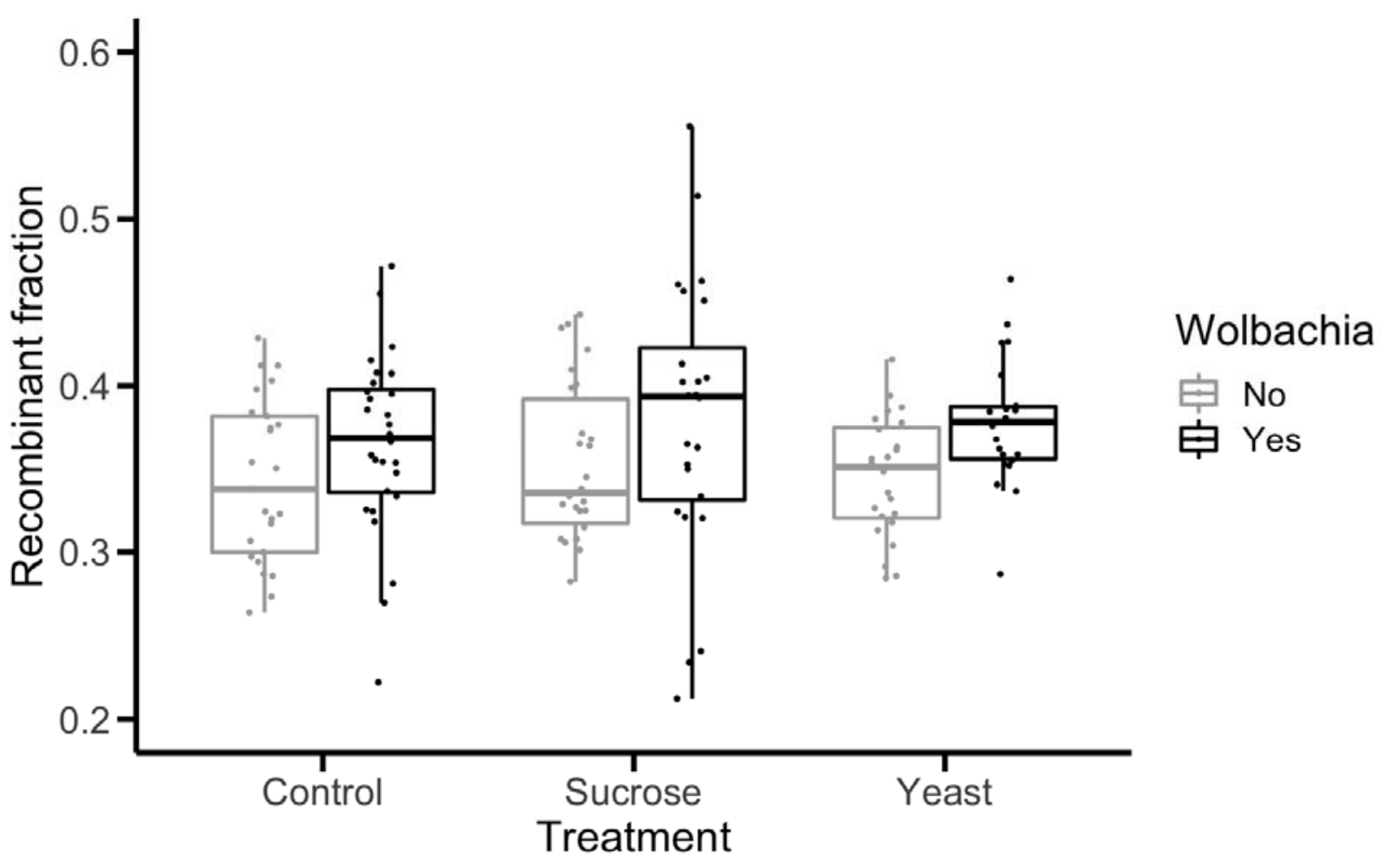

585 Figure 3: Recombination rate, reported as recombinant fraction, of experimental groups.

586 The recombinant fraction is the proportion of recombinant progeny compared to the total number

587 of progeny produced for each cross. Wolbachia-infected flies are shown in black, while

588 uninfected flies are shown in gray. Each point corresponds to the recombinant fraction of a single

589 vial. Boxplots present summary statistics, where the top and bottom edges encompass the first to

590 third quartiles and the middle bar represents the median for each group. Boxplot whiskers extend

591 to the smallest and largest nonoutliers. 


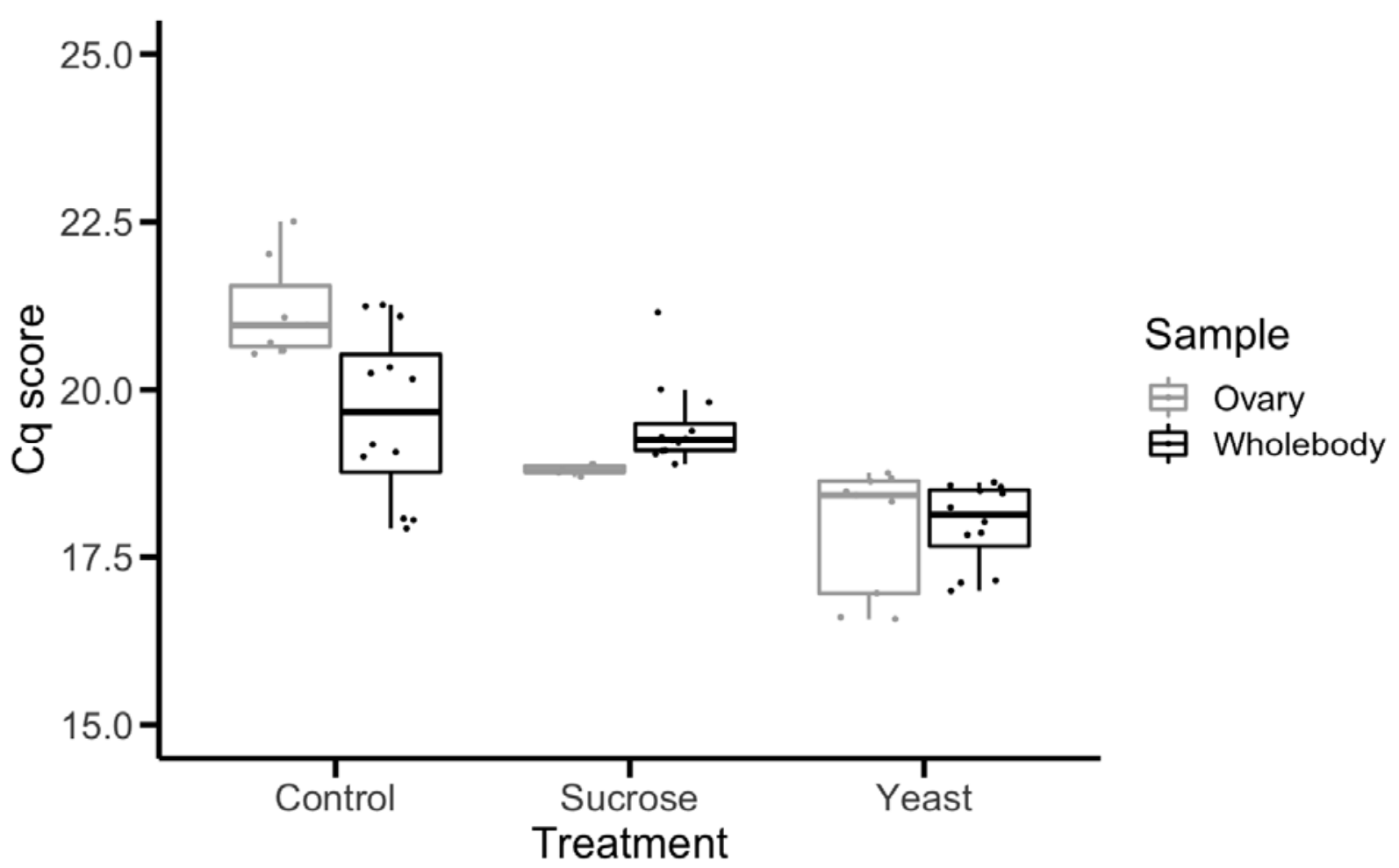

593 Figure 4: Quantitative PCR results from Wolbachia-infected flies in experimental groups.

594 Results are presented as Cq scores, which represent the number of cycles it takes for a sample to

595 reach a threshold amplification level; a lower Cq score corresponds to a higher starting

596 concentration of target DNA in the sample. Both whole-body and ovarian tissue samples were

597 tested for expression of the Wolbachia gene, WSP, and host control gene, Tubulin 2 (not shown

598 here). Each point corresponds to the $\mathrm{Cq}$ score of a single sample. Boxplots present summary

599 statistics, where the top and bottom edges encompass the first to third quartiles and the middle

600 bar represents the median for each group. Boxplot whiskers extend to the smallest and largest

601 nonoutliers. 
TABLES

Table 1: Offspring counts for experimental groups

\begin{tabular}{|c|c|c|c|}
\hline Food treatment & W+ & W- & Total \\
\hline Control & 3284 & 3296 & 6580 \\
\hline Sucrose & 2744 & 2888 & 5632 \\
\hline Yeast & 4952 & 4984 & 9936 \\
\hline Total & 10980 & 11168 & 22148 \\
\hline
\end{tabular}

603

Table 2: Results of general linear model on recombinant fraction

\begin{tabular}{|c|c|c|c|}
\hline Source & DF & L-R ChiSquare & Prob>ChiSq \\
\hline Wolbachia & 1 & 11.315 & $0.0008^{*}$ \\
\hline Food & 2 & 1.723 & 0.42 \\
\hline Wolbachia*Food & 2 & 1.686 & 0.43 \\
\hline Block & 3 & 20.435 & $0.0001^{*}$ \\
\hline
\end{tabular}

604 


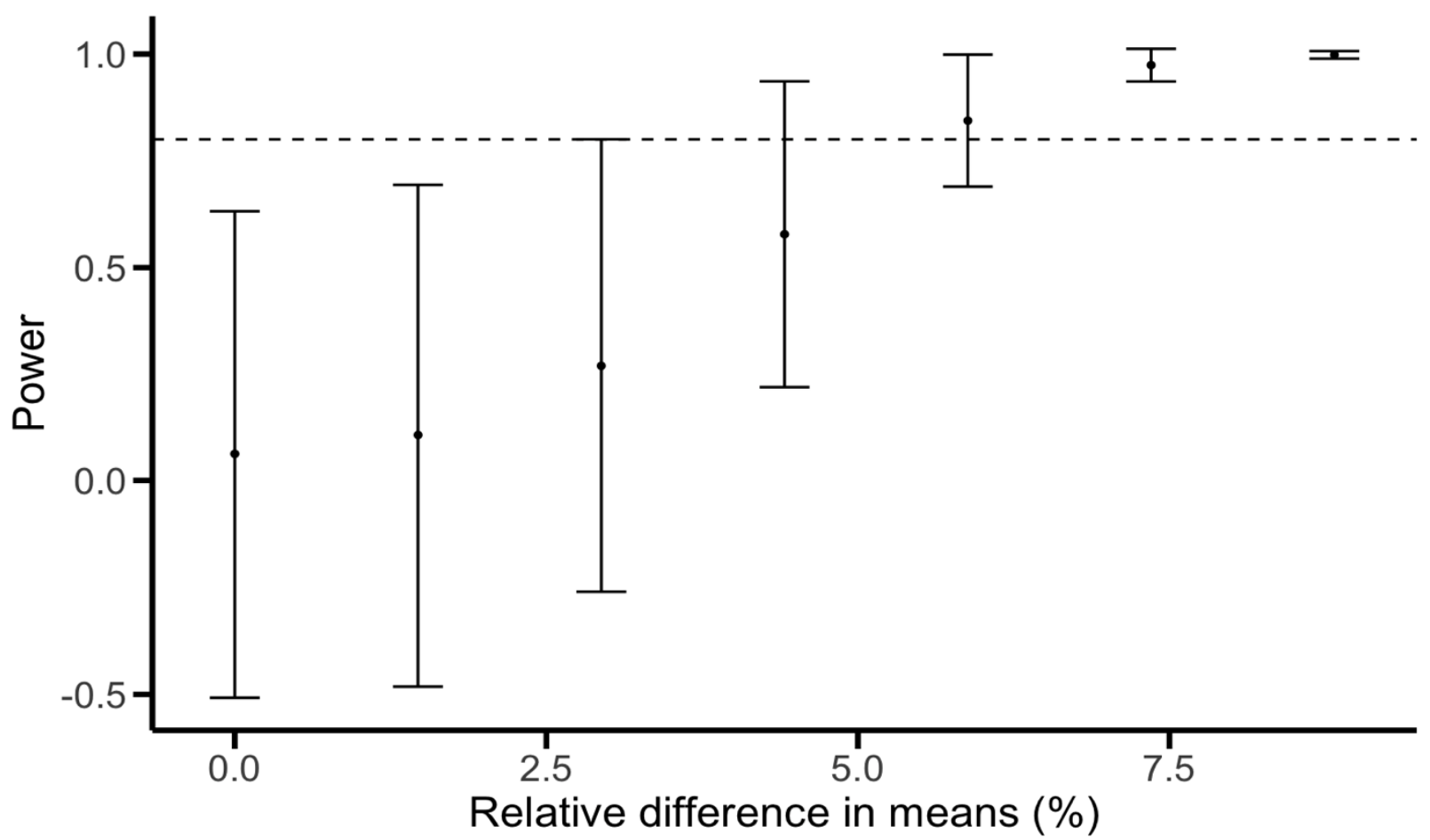

Figure S1: Power analysis to validate recombination results. Experimental data were

608 simulated in $\mathrm{R}$ to create a range of differences in means between experimental groups. These

609 data were then tested using the R package "SIMR", which performed and compiled results from

610 multiple rounds of statistical tests. Results are presented above, where each point corresponds to

611 the power to detect a significant result in simulated data for each effect size and bars represent

$61295 \%$ confidence intervals. The dashed line is set at 0.80 , which is the standard significance

613 threshold for power analyses. 


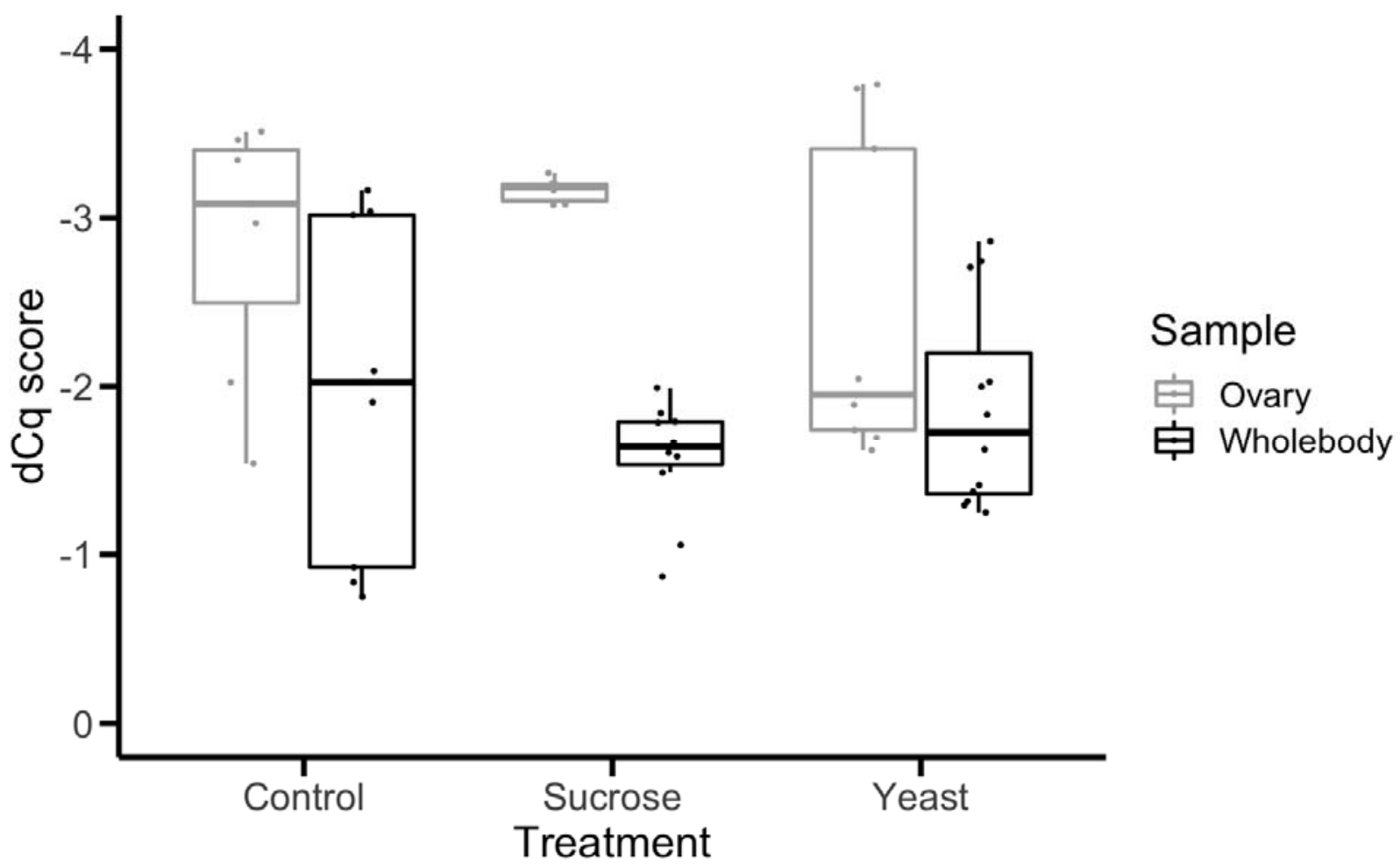

615 Figure S2: dCq scores from Wolbachia-infected flies in experimental groups. Results are

616 presented as $\mathrm{dCq}$ scores, which represent the difference in $\mathrm{Cq}$ score between the Wolbachia

617 gene, WSP, and host control gene, Tubulin 2. A lesser dCq score corresponds to a greater

618 difference between the two genes, which would indicate a higher relative amount of Wolbachia

619 than a sample with a greater $\mathrm{dCq}$ score. Both whole-body and ovarian tissue samples were tested.

620 Each point corresponds to the $\mathrm{dCq}$ score of a single sample. Boxplots present summary

621 statistics, where the middle bar represents the median for each group. 